\section{InterPneu Nürnberg}

5.-6. Oktober 2018

Nürnberg, Deutschland

www.interpneu-nuernberg.de

25. Jahrestagung der Deutschen Gesellschaft für Schlafforschung und Schlafmedizin (DGSM)

11.-13. Oktober 2018

Nürnberg, Deutschland

www.dgsm-kongress.de
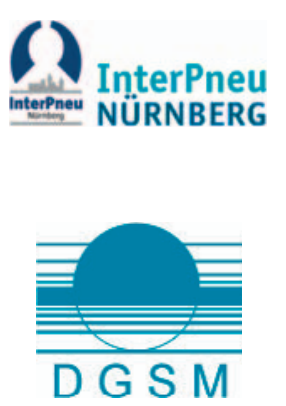

11. Jahreskongress des Tumorzentrums München TZM Essentials 2019

9. Februar 2019

München, Deutschland

www.tzm-essentials.de

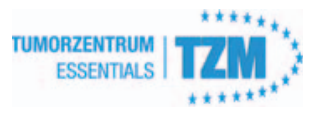

6. Brandenburger Krebskongress

22.-23. Februar 2019

Potsdam, Deutschland

www.mcall-gmbh.de/

bb-krebskongress/

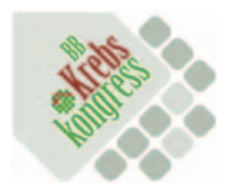

Düsseldorfer Allergie- und Immunologietage 2019

8.-9. März 2019

Düsseldorf, Deutschland

www.duesseldorfer-allergietage.de

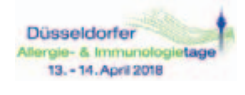

60. Kongress der Deutschen Gesellschaft für Pneumologie und Beatmungsmedizin (DGP) in Kooperation mit der 41. Jahrestagung der Gesellschaft für Pädiatrische Pneumologie

13.-16. März 2019

München, Deutschland

http://pneumologie-kongress.de/

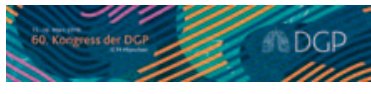

\section{Operation Karriere}

3. November 2018

Berlin, Deutschland

17. November 2018

Köln, Deutschland

7. Dezember 2018

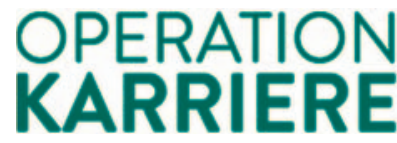

München, Deutschland

www.operation-karriere.de

Masterkurs Thoraxbildgebung im Rahmen des Symposiums "Quo vadis Radiologie?" der Deutschen Gesellschaft für Pneumologie und Beatmungsmedizin (DGP) und der

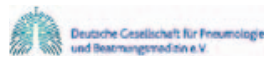
Deutscher Röntgengesellschaft (DRG)

8.-10. November 2018

Hannover, Deutschland

DEUTSCHE RONTGENGESEUSCHAFT

https://pneumologie.de/fileadmin/

user_upload/ 2018-11_Programm_QuoVadis_

2018_0205_final.pdf

11. Deutsches Infektiologie-Update

7.-9. Dezember 2018

Hamburg, Deutschland

ifi.

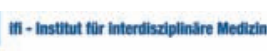

Frühjahrstagung der Arbeitsgruppe Pädiatrie der Deutschen Gesellschaft für Schlafforschung und Schlafmedizin e. V. (DGSM) und der AG Schlafmedizin und Schlafforschung der Österreichischen Gesellschaft 22.-23. März 2019

Wien, Österreich

www.dgsm-paediatrie.de

Humor auf Rezept? Die Dosis bringt den Erfolg! Leipzig, Deutschland

15.-16. Mai 2019

http://www.arztmithumor.de/

veranstaltungen/51/humor-auf-rezept-

die-dosis-bringt-den-erfolgkopie/

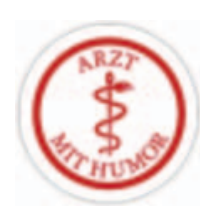

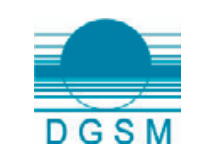

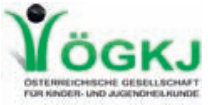

OGKJ für Kinder- und Jugendheilkunde (ÖGKJ) 2nd Cologne Conference on Lung Cancer

26.-27. Juni 2019

Köln, Deutschland

www.cologne-clc.com

www.infektiologie-update.de

\title{
KARGER
}

(c) 2018 S. Karger GmbH, Freiburg 
Jahrestagung der Westdeutschen Arbeitsgemeinschaft für Pädiatrische Pneumologie und Allergologie WAPPA

9.-10. November 2018

Hamm, Deutschland

http://www.wappaev.de/

36. Allergiesymposium der Norddeutschen Arbeitsgemeinschaft für Pädiatrische Pneumologie und Allergologie (NAPPA)

16.-17. November 2018

Lübeck, Deutschland

www.nappa-ev.de

18. Pneumologie-Update-Seminar

16.-17. November 2018

Wiesbaden, Deutschland

23.-24. November 2018

Berlin, Deutschland

https://pneumo-update.com/

\section{Deutsche Mukoviszidose Tagung}

22.-24. November 2018

Würzburg, Deutschland

https://www.muko.info/angebote/veranstaltungen/deutsche-

mukoviszidose-tagung-dmt/

\section{ELCC 2019 - European Lung Cancer Congress}

10.-13. April 2019

Genf, Schweiz

www.esmo.org/Conferences/ELCC-2019-European-Lung-Cancer-

Congress 